\title{
TRAJECTORY ESTIMATION STUDIES FOR LONG COASTING PHASE OF MARS MISSION
}

\author{
V. Seshagiri Rao ${ }^{1}$, D. Narayana Rao ${ }^{2}$ \\ ${ }^{1}$ Satish Dhawan Space Centre SHAR, Sriharikota, India \\ ${ }^{2}$ SRM University, Chennai, India
}

\begin{abstract}
At Sriharikota range, configuration of down range tracking network, Real-time tracking and trajectory estimation play a critical role during a satellite launch for flight safety as well as mission monitoring. Criticality is more when the mission parameters vary during the launch window to meet the mission requirements. PSLV-C25, the $320 \mathrm{~T} X L$ version is intended to inject the 1337.24 Kg MARS Orbiter into $250 \mathrm{Km} * 23500 \mathrm{Km} * 19.2 \mathrm{deg}$ Sub-GTO orbit. MARS Orbiter Mission [MOM] is the first Indian interplanetary mission to orbit a spacecraft around MARS in an elliptical orbit of $360 \mathrm{~km} * 80000 \mathrm{~km}$. This paper deals with the trajectory estimation and prediction methodologies studied and established at Sriharikota Range for this launch. The major challenge in the Mars orbiter mission is to configure the Down range network of Telemetry stations in view of large variation in the Argument of Perigee (AOP) requirement ranging from $276^{\circ}$ to $289^{\circ}$ during injection over the period of launch window. Requisite AOP facilitates transit of the Spacecraft from Earth to Mars using minimum energy Hohmann transfer. The change in the requirement of AOP each day in turn demanded a new trajectory with its characteristic changes in long coasting duration, fourth stage ignition time and subsequent MARS Orbiter injection time into sub-GTO. It is mandatory to capture the Telemetry data during those critical events to assess the success of the mission. The flight duration was around 3000s and the coasting duration was 1600s before the ignition of the PS4 stage. Study of configuration of mobile Telemetry stations on Shipborne terminals is carried out to cater to visibility requirements of critical events such as fourth stage ignition time and subsequent MARS Orbiter injection. State vector accuracy studies are carried out for the Ship-borne radar data of the vehicle during long coasting using Linear Kalman filter. Also a trajectory extrapolation algorithm is designed and studied to provide extrapolated trajectory during the long coasting period after $3^{\text {rd }}$ stage burn out which in turn is used to compute and display trajectory parameters to mission executives and provide antenna-pointing information to the ship-borne terminals. This paper presents the trajectory estimation methodology proposed, extrapolation techniques adopted and accuracies achieved for the long coast duration.
\end{abstract}

Keywords: Mars Orbiter Mission (MOM), Flight Safety, Trajectory estimation, Network simulations, Argument of Perigee (AOP).

\section{INTRODUCTION}

Configuration of down range tracking network, Real-time tracking and trajectory estimation play a critical role during a satellite launch catering to flight safety as well as mission monitoring[1]. This demands design and development of a robust Real time system [RTS] consisting of down range tracking and telemetry network to meet the functional and performance requirements for launch operations. To meet the mission requirements at Sriharikota, the space port of India, there is established a long range tracking network consisting of $4 \mathrm{C}$-Band radars and two SBand radars. For the MARS orbiter mission, the challenge was to design a real time system which can provide continuous data flow during the long coast duration due to the requirement of higher value of Argument of Perigee (AOP) and Right Ascension of Ascending Node (RAAN). The existing network of telemetry stations caters to $1600 \mathrm{sec}$ of trajectory visibility. To meet the additional real time requirement of long coasting, two ship-borne terminals were located at a pre-designated locations in the Pacific Ocean to capture the fourth stage ignition and spacecraft separation events. The Day to day variation in the AOP requirement demanded varying ship positions such that the PS4 ignition and spacecraft separation events are captured. As standby arrangement, two Brazilian ground stations at Cuiaba and Alcantara were also hired to acquire these events in the post-injection period through onboard stored data.

To have continuous real time display during the non visibility period, a trajectory extrapolation module [TRAJEX] was designed and developed to compute the extrapolated trajectory for display to the mission executives in the Mission Control Centre [MCC]. Accuracy of the extrapolated trajectory was assessed by comparing it with filtered state vector of the vehicle tracked by a precision radar onboard the ship terminal. A C-band radar is proposed to track the vehicle in coasting phase as well as in thrusting phase. Trajectory estimation by a Linear Kalman filter is studied and the filtered state vector is compared against its theoretical trajectory as well as extrapolated trajectory to assess its accuracy. 


\section{REAL TIME SYSTEM FOR MARS ORBITER MISSION}

Mars Orbiter Mission is ISRO's first interplanetary mission with a spacecraft designed to orbit Mars. The launch vehicle is monitored in real time by the mission executives to evaluate its performance. Tracking systems and telemetry systems at the range as well as down the range provide the trajectory information and vehicle sub-systems' health for performance evaluation.

The Real Time system for Mars Orbiter Mission consists of dual processing chains. Each chain is configured with a server, which receives data from all the tracking and telemetry sources, processes and transmits onto the network[2]. The tracking data and the telemetry data are acquired at $10 \mathrm{~Hz}$ and $2 \mathrm{~Hz}$ respectively, processed and logged. Figure. 1 depicts the configuration of range safety Real time system.
The processing software edits and filters the tracked data. The telemetry data is converted into engineering units as per data formats and is scaled. Kalman filter is used to estimate the state vector from each tracking source. Trajectory display parameters like Position, velocity and on-board systems' performance data are computed. Each graphic station displays processed data from all tracking and telemetry sources at each instant. The antenna pointing information for various tracking and telemetry stations including Port Blair, Brunei, Biak, Ship-borne terminals OP1 and OP2 is derived from a single selected source based on priority logic. The telemetry parameters to be displayed on flight parameter display are taken from the valid sources.

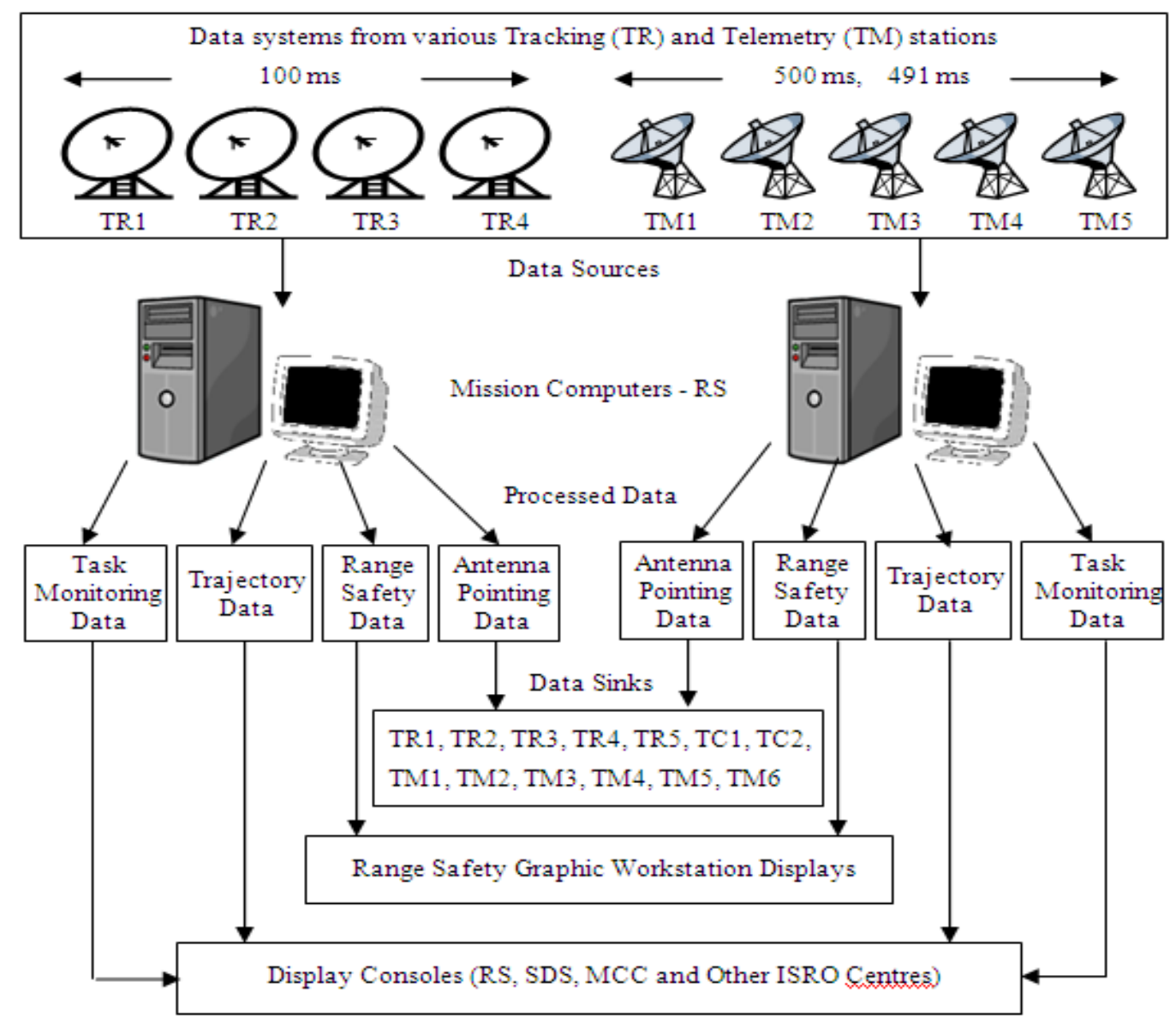

Fig.1 Block Diagram of Real time system

After an exhaustive study and analysis, extrapolated state vector from $\boldsymbol{f}$ and $\boldsymbol{g}$ series is used for generating trajectory and antenna pointing information during the long visibility gap. On chain-1 INS-based extrapolated state vector is used and on chain - 2 GAINS-based extrapolated state vector is used. For Mission Control Centre displays INS-based extrapolated state vector is used.

\section{REAL TIME MISSION REQUIREMENTS}

The major challenge encountered in the PSLV C-25 mission is to provide continuous flow of data to the mission executives for the long coast duration of 1600 s which was never encountered before. This was mainly due to the Optimal departure of satellite from EPO [Earth parking orbit] to TMO [Trans MARS Orbit] requiring large 
Argument of Perigee (AOP) value. PSLV trajectory design demands long coast duration of the order of 1600s between third stage burnout to Fourth stage ignition to meet the AOP constraints.

Due to long coast duration, additional ship-borne terminals are required for vehicle telemetry. All these constraints posed a major challenge for the designers to design a robust real time system which can provide for real time monitoring. To accomplish the above challenging tasks in real time and near real time, the following strategies and services were evolved.

- a). Study of Linear Kalman filter for processing the ship-borne radar data during coasting phase and PS4 thrusting phase. Process noise for this period is computed using the technique of model compensation using Ideal state estimation.

- b).Development of trajectory extrapolation module using $\mathbf{f}$ and $\mathbf{g}$ series. This extrapolated state vector is used for trajectory computation and antenna pointing information to tracking stations.

- c). Locating the Ship borne Terminal OP1 and OP2.

\section{LINEAR KALMAN FILTER}

The well known and widely used Kalman filter is 40 years young and it has been exploited for many applications ranging from putting man on the moon to monitoring fuel efficiency of a car to alert mechanical degradation using a personal computer. In this chapter the basic equations of Kalman filter are given with the assumptions used to obtain them. Given the dynamics, the measurement equations and other related parameters and measurements up to time $T_{K}$ the filtering problem is to find the best optimal estimate in the sense linear, unbiased and minimum variance. A minimum variance unbiased estimate has the property that its error variance is less than or equal to that of any other estimate.

\section{Kalman Filter structure}

The predicted state using the State Transition matrix and previously estimated state

$$
\mathrm{X}_{\mathrm{K}}=\Phi_{\mathrm{K} / \mathrm{K}-1} \quad \mathrm{X}_{\mathrm{K} / \mathrm{K}-1}
$$

Predicted measurement using predicted state and Observation matrix is

$$
\mathrm{Z}_{\mathrm{K} / \mathrm{K}-1}=\mathrm{H}_{\mathrm{K}} \quad \mathrm{X}_{\mathrm{K} / \mathrm{K}-1}
$$

Covariance of the predicted state is

$$
\mathrm{P}_{\mathrm{K} / \mathrm{K}-1}=\Phi \mathrm{k} \quad \mathrm{P}_{\mathrm{K}-1 / \mathrm{K}-1} \Phi_{\mathrm{K} / \mathrm{K}-1}^{\mathrm{T}}+\mathrm{Q}_{\mathrm{K}-1}
$$

Kalman gain is computed using predicted state covariance, measurement noise matrix and Observation matrix

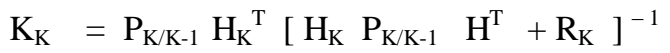

State estimate from actual measurement is

$$
X_{K / K}=X_{K / K-1}+K_{K}\left(Z_{K}-Z_{K / K-1}\right)
$$

Covariance of the estimated state is

$\mathrm{P}_{\mathrm{K} / \mathrm{K}}=\left[\begin{array}{lll}\mathrm{I}-\mathrm{K}_{\mathrm{K}} & \mathrm{H}_{\mathrm{K}}\end{array}\right] \mathrm{P}_{\mathrm{K} / \mathrm{K}-1}$

By knowing the initial state $X_{0}$ and its covariance $P_{0}$, state process noise $\mathrm{Q}$, measurement noise $\mathrm{R}$ the first four steps can be executed using the equations 1-4. As soon as measurements are available last two equations 5 and 6 can be used to estimate the state and its covariance. The cycle is repeated for the next measurements.

\subsection{Trajectory Extrapolation during Coasting}

\section{Phase using $f$ and $g$ Series}

Precise determination of the launch vehicle performance in realtime is through the telemetry data acquired from various telemetry stations located on the launch site and down the range. It is useful for monitoring the performance of the launch vehicle, health of the satellite and also useful in determining the preliminary orbit of the spacecraft. But for certain missions there exists certain constraints which result from imposing physical limitations on trajectory or earth orbit, where the telemetry ground stations cannot provide the launch vehicle performance for the entire mission till injection of the satellite. In order to determine the trajectory of such missions, $\boldsymbol{f}$ and $\boldsymbol{g}$ series predictor algorithm can be used to provide the rapid trajectory computation of the vehicle. Launch vehicle trajectory can be determined based the expansion of the solution of the Newton's laws into Taylor series, known in celestial mechanics as the $\boldsymbol{f}$ and $\boldsymbol{g}$ power series. These power series can be used to integrate the equations of the motion of bodies and obtain trajectory from the initial position and velocity when the body is purely under the influence of acceleration due to gravity and atmospheric drag[3].

The particle in the $3 \mathrm{D}$ is considered. The equation of the motion for a particle in $3 \mathrm{D}$ is given by the following expression

$$
\mathrm{R}=\left[\begin{array}{l}
x \\
y \\
z
\end{array}\right]
$$

where $\mathrm{r}$ is given by the expression $\mathrm{R}=\sqrt{\left(x^{2}+y^{2}+z^{2}\right)}$

So, the $3 \mathrm{D}$ position of the particle at each time instant can be written as

$$
r_{t}=\left[\begin{array}{l}
x_{t} \\
y_{t} \\
Z_{t}
\end{array}\right]
$$


The $3 \mathrm{D}$ velocity of the particle is given by

$$
\mathrm{V}=\frac{d r}{d t}=\dot{r}=\left[\begin{array}{c}
\dot{x} \\
\dot{y} \\
\dot{z}
\end{array}\right]=\left[\begin{array}{c}
V_{X} \\
V_{Y} \\
V_{Z}
\end{array}\right]
$$

The acceleration of the particle is given by

$$
\mathrm{a}=\frac{d v}{d t}=\ddot{r}=\left[\begin{array}{c}
\ddot{x} \\
\ddot{y} \\
\ddot{z}
\end{array}\right]=\left[\begin{array}{l}
a_{x} \\
a_{y} \\
a_{z}
\end{array}\right]
$$

The generalized equation of the $\mathrm{f}$ and $\mathrm{g}$ series is given below

$$
r_{1}=\mathbf{f}(T) r_{o}++g(T)\left(\frac{d r}{d t}\right)
$$

Where $\mathrm{r}$ is the position, $T=\left(\mathrm{t}_{1}-\mathrm{t}_{0}\right)$, $\mathrm{f}$ and $\mathrm{g}$ are the power series. If the position and the velocity $\left(1^{\text {st }}\right.$ derivatives of the position) components at $t_{0}$ are known then the $2^{\text {nd }}, 3^{\text {rd }}$ and $4^{\text {th }}$ derivatives of the position components at $\mathrm{t}_{0}$ are computed. Substituting the $2^{\text {nd }}, 3^{\text {rd }}$ and $4^{\text {th }}$ derivatives of position components at $t_{0}$ the position and velocity components at time $t_{1}$ is obtained. This computation is continued till the end of coasting phase. For the Mars Orbiter Mission, the coasting period starts at third stage burn out and continues up to fourth stage ignition. The fourth stage ignition was around 2005s on the launch day of $05^{\text {th }}$ November 2013. State Vector is extrapolated at $10 \mathrm{~Hz}$ up to $2005 \mathrm{~s}$ using $f$ and $g$ series. Figure. 2 gives the real time and extrapolated trajectory for MARS mission

\subsection{Ship - Borne Terminals for Telemetry Acquisition}

Two ship - borne terminals were used in real time to meet the long coast duration constraint imposed by large AOP requirement. It was a challenging exercise for the range team in finalizing the suitable designated locations for the ships so as to acquire real time information on Fourth stage ignition and spacecraft separation. Every day the trajectory was changing to meet the required AOP. Range team carried out extensive trajectory simulations to determine the visibility of these ships at various locations, such that shipborne radars can acquire and track the vehicle and shipborne telemetry can provide the tracking telemetry data of fourth stage ignition and MOM separation to mission executives. "All begins well will end well", this exercise by range team was fruitful in arriving at vantage locations for ships based on sea roughness.

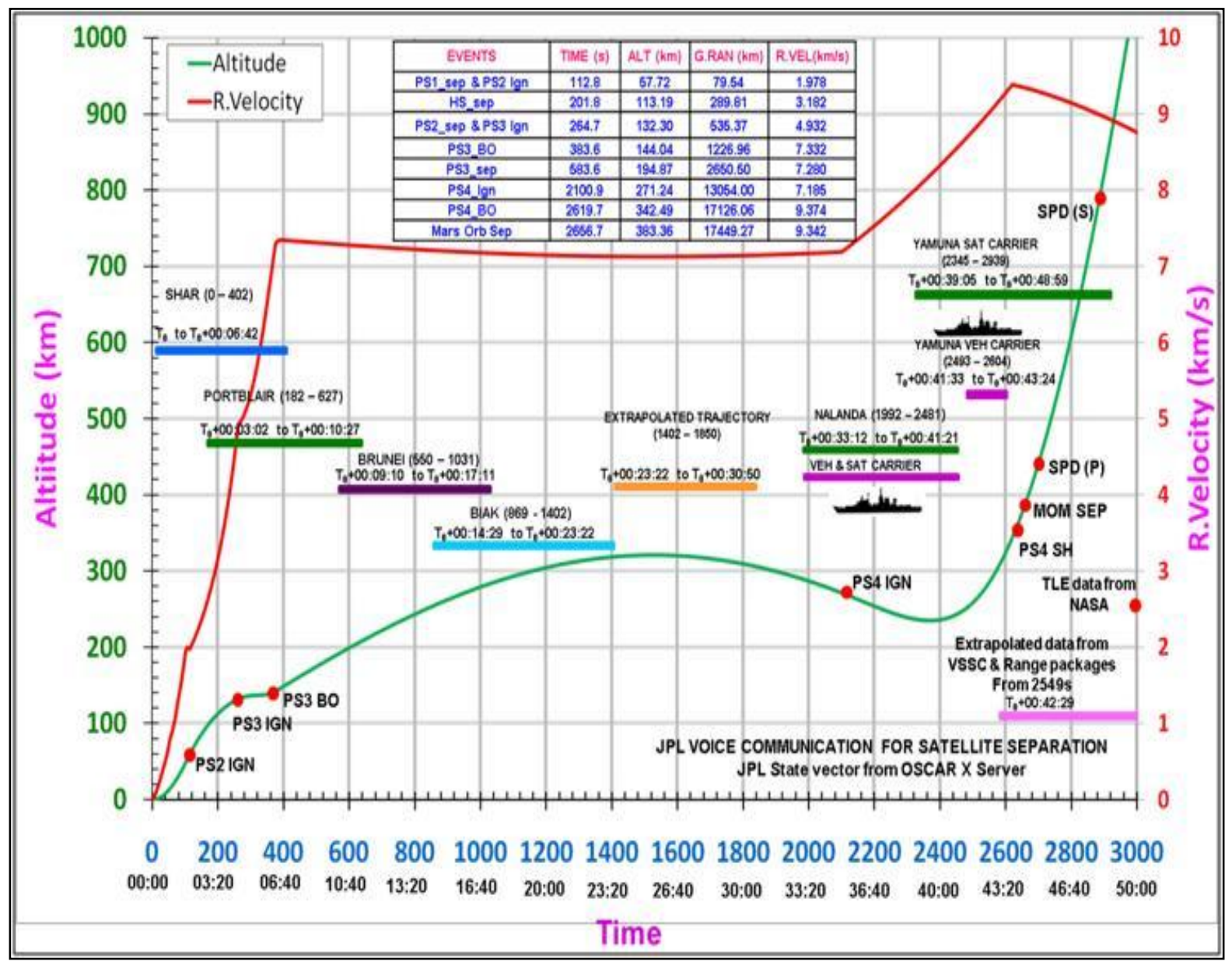

Fig 2 Real time and extrapolated trajectory for MARS mission 


\section{RESULTS AND DISCUSSION}

For study the tracking accuracies of ship-borne radar are considered as $10 \mathrm{~m}$ in Slant range, and 1 milli rad in angles. Process noise is generated by the authors using the established technique of model compensation by ideal state estimation. Tracking data is generated at $10 \mathrm{~Hz}$ w.r.t the ship-borne radar and is processed using Linear Kalman filter. The errors in the tracking parameters are plotted against their theoretical bounds are plotted in Figure. 3 to Figure.5. The position error and velocity error are plotted in Figure.6. The errors in the tracking parameters are within their theoretical bounds. This deduction amply establishes the fact that Linear Kalman filter with process noise as model compensation by ideal state estimation provides optimal state estimate of the vehicle during long coasting also. The state vector accuracy in terms of position error and velocity error are within $10 \mathrm{~m}$ and $10 \mathrm{~m} / \mathrm{sec}$ except at time of the time of closet approach. This fact proves the suitability of Linear kalman filter for coast phase deployment. Also f and $\mathbf{g}$ algorithm could provide extrapolated trajectory for display as well as antenna pointing information to the ship-borne terminals. This aided the ship-borne terminals to acquire the critical events such as PS4 ignition and MOM separation.

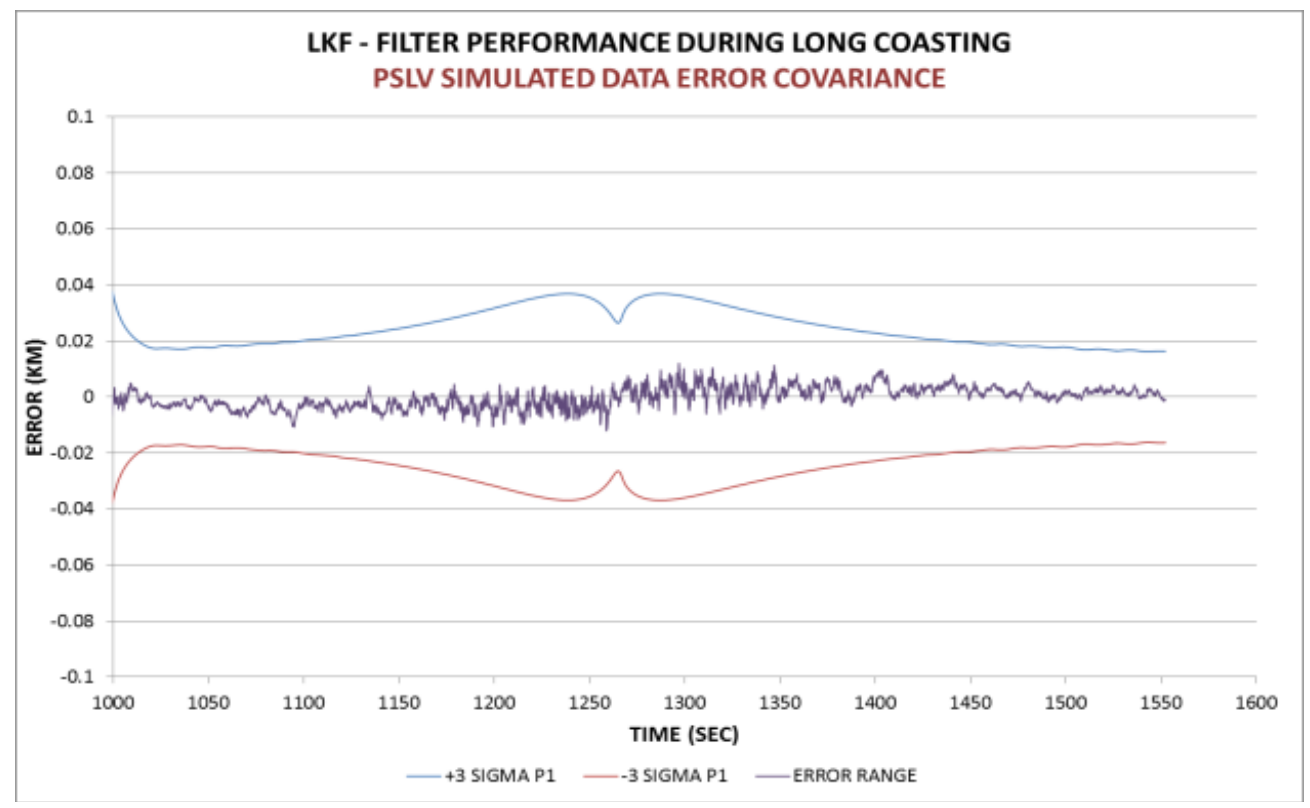

Fig 3 Error in Slant range against its $3 \sigma$ limits

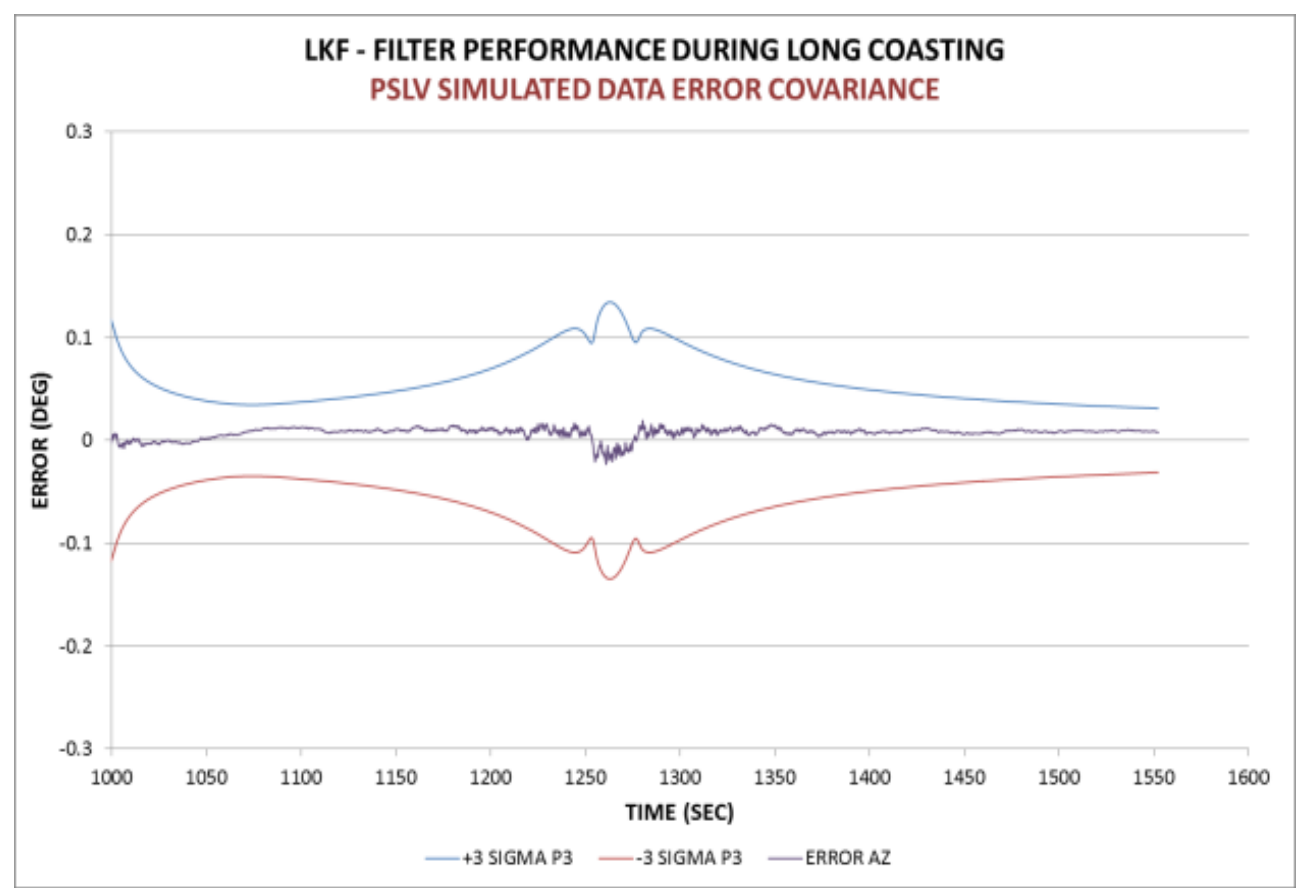

Fig 4 Error in Azimuth against its $3 \sigma$ limits 


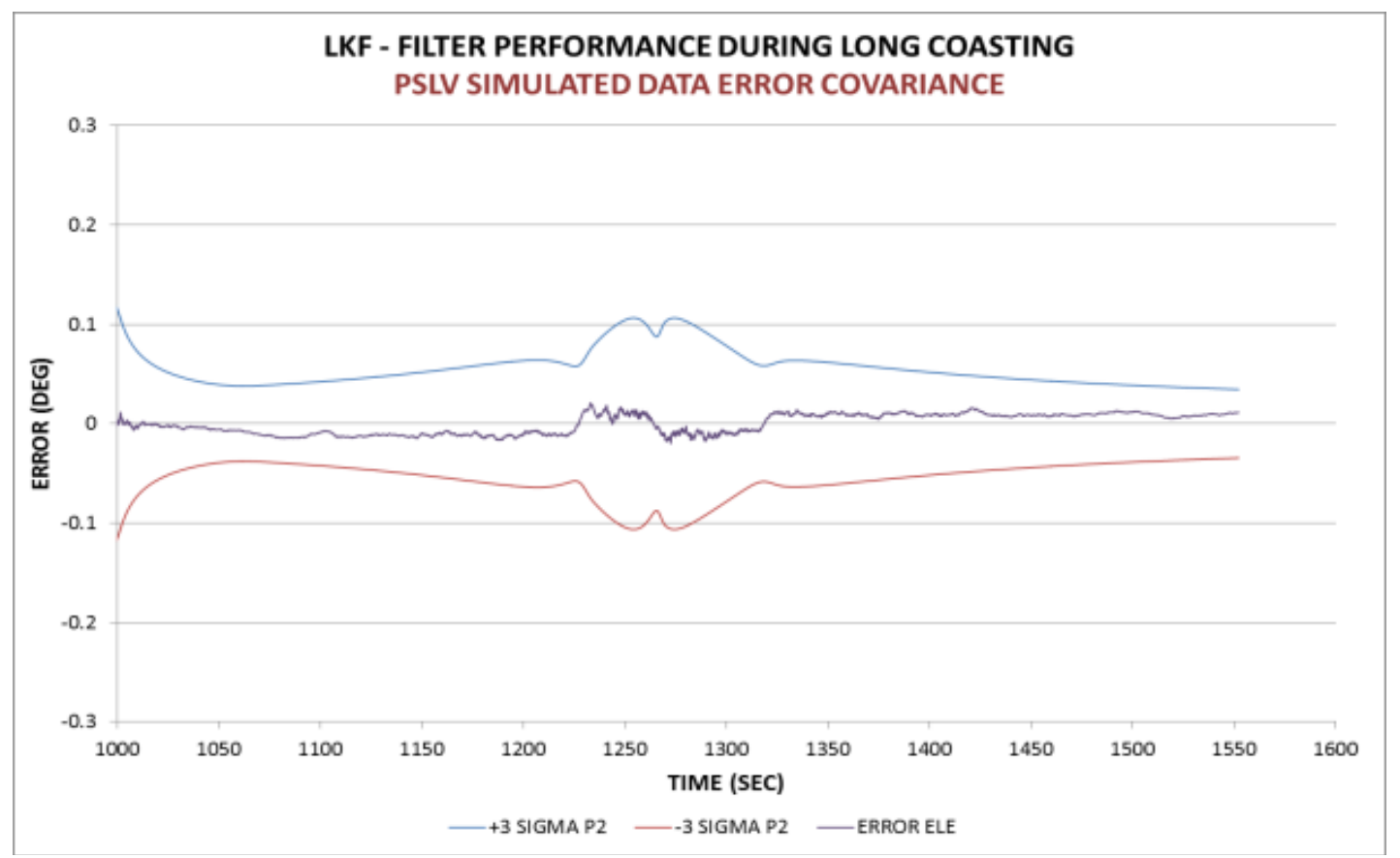

Fig 5 Error in elevation against its $3 \sigma$ limits

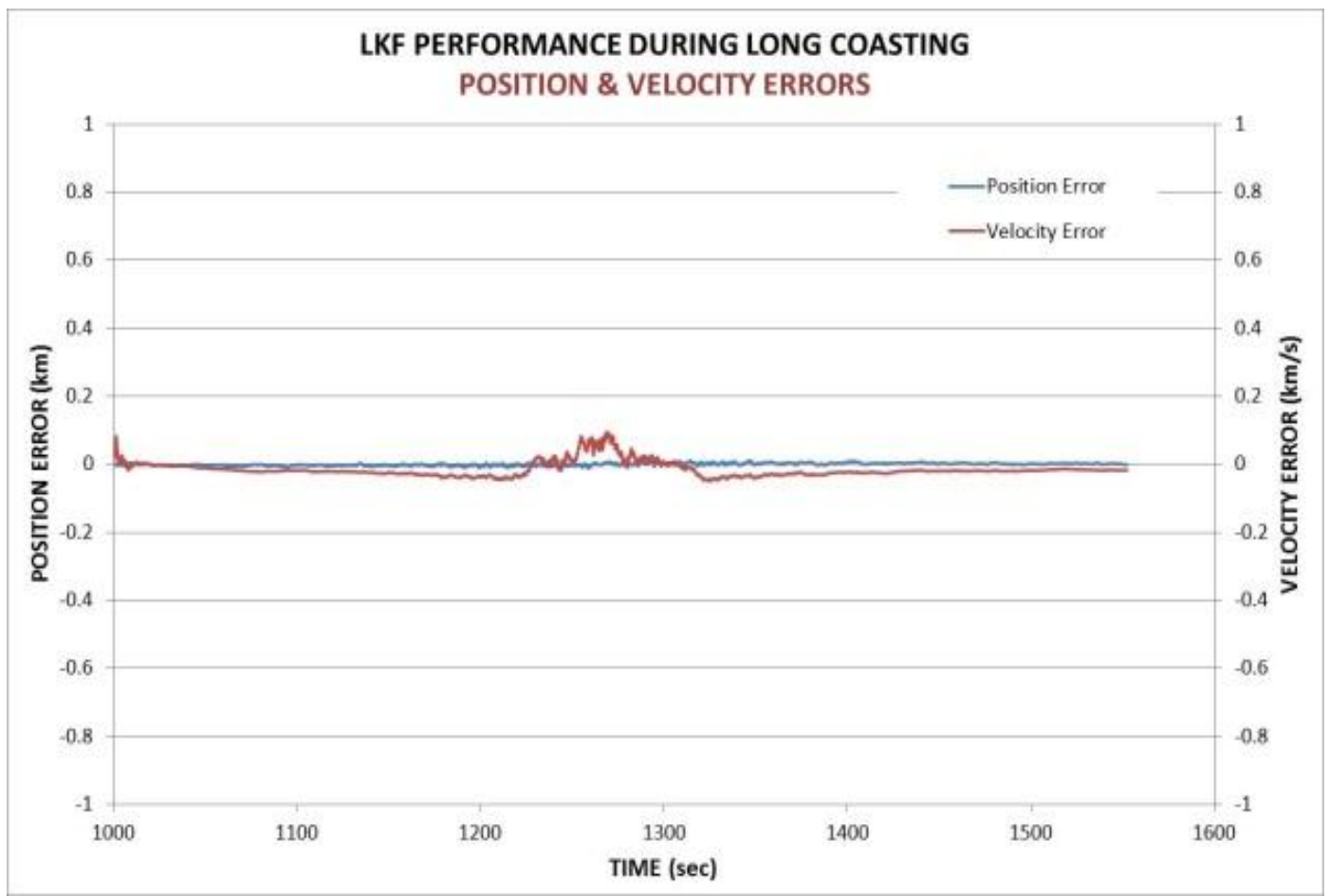

Fig 6 Position and velocity errors for LKF with easurements $1 \sigma$ noise

\section{CONCLUSION}

Various mission constraints imposed by the MARS orbiter mission on the Real time systems were met by incorporating validated trajectory estimation and extrapolation algorithms. The Range team also came out with a definitive solution to locate the ships in pre designated locations. The modules developed by the range safety team provided vital inputs in configuring the real time systems for the mission. All the mission-critical requirements of PSLV-C25/MARS Orbiter mission were met by Range real time systems[4].

\section{ACKNOWLEDGEMENTS}

The authors wish to thank Dr. MYS Prasad, Director SDSC SHAR for granting permission to publish this paper. Success in this critical area would not have been possible but for the dedicated support and unstinted cooperation of colleagues in Range Operations at SDSC SHAR. 


\section{REFERENCES}

[1] Flight Safety Plan for PSLV-C25/Mars Orbiter Mission by Flight safety team, ISRO-SHAR-04-CTR-11-2013

[2] PJC Reddy, P Sunil, R Varaprasad and V Seshagiri Rao "Reliability Practices for Real Time Systems at SDSC SHAR", Presented at ICPAP 2011, Bangalore, 2011

[3] KM Dinesh babu et al, "Trajectory Extrapolation using $f$ and $g$ series for MARS orbiter mission". ISRO-SHAR-04-C-TR-10-2013

[4] R.Gnanasambanthan et al, "PSLV-C25/MARS orbiter Mission: Post flight data analysis of tracking and telemetry data" ISRO-SHAR-03-R-TR-10-2014 